\title{
Slide-Free Microscopy via UV Surface Excitation
}

Richard Levenson ${ }^{1}$, Farzad Fereidouni ${ }^{1}$, Zachary Harmany ${ }^{1}$, Miao Tan $^{1}$, Mirna Lechpammer ${ }^{1}$, and Stavros Demos ${ }^{2}$

${ }^{1 .}$ Dept. of Pathology and Laboratory Medicine, UC Davis Medical Ctr., Sacramento, CA.

2. Lawrence Livermore National Laboratory, Livermore, CA.

Microscopic evaluation of tissue remains the definitive diagnostic procedure in the evaluation of most solid tumors, as well as of many other disease processes. Conventional brightfield or fluorescence microscopy works best with thin, stained specimens mounted on glass slides, but to prepare these requires hours of processing and the help of highly skilled technical personnel. We describe a new, inexpensive form of light microscopy, based on UV surface excitation (MUSE), that can generate highquality histology and histopathology images directly from cut surfaces of fresh (or fixed) tissue samples of any thickness, with less than 1 minute of preparation. Unlike standard diagnostic processes, it is nondestructive, preserving the specimen for downstream molecular analyses. Preliminary description of this method, which depends on the shallow tissue penetration of sub-300-nm light, can be found in [1].

While alternative microscopy-scale methods, such as confocal, two-photon, or optical coherence tomography approaches [2-6], can generate reasonable images from thick tissues, such instrumentation is typically complex, expensive, and not widely available. With MUSE, thick pieces of tissue obtained by biopsy, surgery or necropsy can be imaged directly, eliminating any requirement for conventional histology processing, formalin-fixation, paraffin-embedding, or thin-sectioning. The method is rapid, and by providing diagnostic-level information in minutes, can potentially change the velocity of clinical encounters; Moreover, MUSE can be more informative than conventional histology, because it provides information on surface topography, typically effaced by thin-sectioning procedures.

Figure 1 illustrates the basic MUSE configuration, which is based on an inverted microscope design, largely because it is the easiest way to position large, variably shaped tissue specimens. The sample rests on (or is lightly compressed against) a UV-transmitting flat optical surface, typically quartz or sapphire (replacing the coverslip in conventional microscopy). The excitation light is provided by one or more UV-emitting LEDs with maximum emission wavelength centered around 275-285 nm, arranged to illuminate the sample in oblique configuration. We typically use a $10 \mathrm{X}$ lens and a scientific-grade 9Mpixel color (RGB) CCD camera.

Samples need only be cut with a sharp knife or scalpel to provide a flat imaging surface, and then briefly stained. A convenient dye combination includes rhodamine and Hoechst in phosphate-buffered saline. The specimen is dipped into this mixture for $10 \mathrm{~s}$ and then washed gently in more PBS, and then can be immediately positioned on the UV-transparent stage for imaging. Depending on the specimen and the brightness of the LED(s) used, images can be acquired in as little as $100 \mathrm{~ms}$ per field. Multiple fields can be taken by translating the stage, and then montaged into a single, large field-of-view final image file.

MUSE images are acquired in fluorescence and take advantage of the fact that many fluorescent dyes can all be excited at $280 \mathrm{~nm}$. The resulting images display colors unfamiliar to pathologists used to the pink and blue seen in transmission views of hematoxylin- and eosin- (H\&E) stained specimens. Fig. 2 (left) demonstrates the appearance of prostate tissue liver stained with rhodamine and Hoechst. The 
orange background is different from what is usually seen, but on the other hand, the fact that MUSE can image thick pieces of tissue, rather than thin-cut slices provides an opportunity to appreciate the surface profile. Here it is clear that the large prostate ducts are conduits leading into the plane of the tissue. If however, it is important to reproduce H\&E-like appearance, it is possible to map the acquired data into a transmission-like color space, as shown in Fig. 2, right, through various expedients. One of the most simple is to take the red and blue channels of the RGB output of the original fluorescence image, invert them, and re-combine using simple Photoshop tools. This approach is easy to implement in real time, so that the monitor can display a live, re-mapped H\&E-like image directly from the fluorescence raw camera output. Alternative, more sophisticated color-mapping methods are also available.

We have described a novel approach to rapid microscopic imaging of slide-free tissue specimens that may be able to replace frozen section methods for rapid intra-operative guidance, provide novel surface topography information, accelerate the diagnostic process, preserve tissues for subsequent molecular assays, and make available tissue diagnostics in underserved and remote environments.

\section{References:}

[1] F Fereidouni et al, Proc. SPIE. (2015) 9318: 93180F

[2] D. Kang et al, Biomed Opt Express. 4 (2013), 41925.

[3] M Gabriele et al, Invest Ophthalmol Vis Sci 52 (2011), 2425.

[4] Y Tao et al, Proc Natl Acad Sci USA 111 (2014), 5304.

[5] J Durkin et al, Dermatol Surg 40 (2014), 266.

[6] J Bini et al, J Biomed Opt 16 (2011), 076008.

[7] The authors acknowledge funding from a UC Davis STAIR grant and from UCD Pathology Dept.

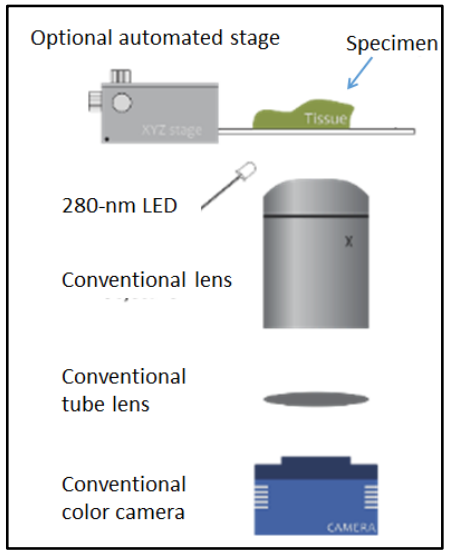

Figure 1. Optical design of the MUSE microscopy system
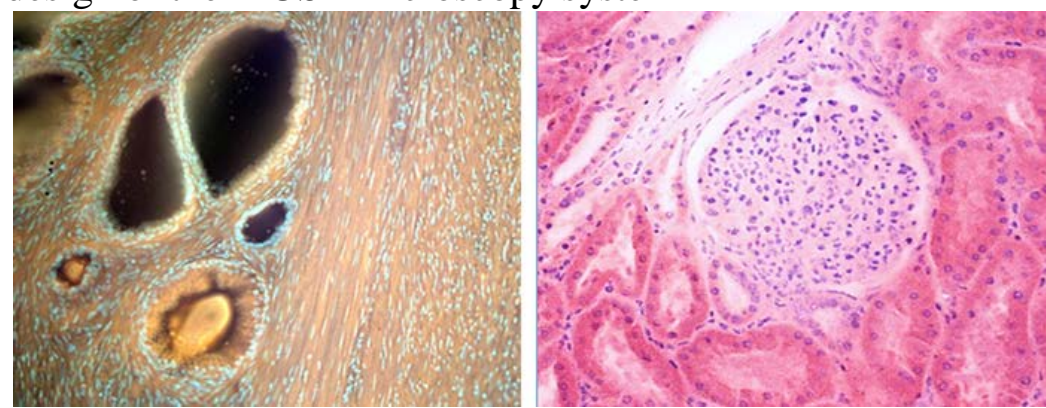

Figure 2. Examples of MUSE images. Left: prostate, fluorescence. Right, renal tissue, pseudo-H\&E 